\title{
ARTIGOS
}

Submetido 25.02.2014. Aprovado 25.11.2014

Avaliado pelo processo de double blind review. Editor Científico: Tales Andreassi

DOI: http://dx.doi.org/10.1590/So034-759020150509

\section{PROPOSTA DE INVESTIGAÇÃO SOBRE O USO DE SOFTWARE NO SUPORTE À INOVAÇÃO EM SERVIÇOS}

\author{
Investigation proposal on software as a tool for innovation in services \\ Propuesta de investigación sobre software como soporte a la innovación en \\ servicios
}

\begin{abstract}
RESUMO
Este artigo examina os objetivos, instrumentos e características das inovações habilitadas por Tecnologias da Informação e Comunicação (TICS) no Brasil, enfatizando as tecnologias utilizadas, os canais habilitados pelas ferramentas digitais, os processos de aprendizado para inovação e as mudanças organizacionais necessárias para o uso de ferramentas avançadas. A metodologia consiste na adaptação de taxonomias existentes para os propósitos específicos da pesquisa e a realização de uma pesquisa de campo com o auxílio do software Atlas.ti. Os resultados mostram que as inovações habilitadas por TICs não constituem eventos isolados e pontuais, uma vez que alteram processos, formas de relacionamento com parceiros e clientes, e requerem uma maior integração de funções organizacionais. Importantes diferenças foram observadas em função do porte da empresa, da internacionalização das operações, da capacitação técnica e do grau de acesso a ferramentas proprietárias.
\end{abstract}

PALAVRAS-CHAVE | Tecnologias da Informação e Comunicação, gestão, inovação, software, serviços.

\section{ABSTRACT}

This article examines the goals, instruments and characteristics of Information and Communication Technology (ICTs) enabled innovations in the Brazilian software industry emphasizing technologies, transmitting channels, learning processes, and organizational changes required by advanced digital tools. The methodology steams from the adaptation of an existing taxonomy for our specific purposes and the development of a field research in Brazil, with the help of the software Atlas.ti. We found that ICTs enabled innovations are not isolated events, since they affect existing processes, the relationship with clients and suppliers, and require greater integration of organizational functions. Important differences were observed due to the firms' size, internationalization level, technical capabilities and access to proprietary technology.

KEYWORDS I Information and Communication Technology, management, innovation, software, services.

\section{ALESSANDRO DE ORLANDO MAIA PINHEIRO}

alessandro.pinheiro@ibge.gov.br Doutor em Ciências Econômicas pela Universidade Federal do Rio de Janeiro, Instituto de Economia - Rio de Janeiro - RJ, Brasil

\section{PAULO BASTOS TIGRE}

ptigre@ie.ufrj.br

Professor da Universidade Federal do Rio de Janeiro, Instituto de Economia - Rio de Janeiro - RJ, Brasil

\section{RESUMEN}

Este artículo examina los objetivos, instrumentos y características de las innovaciones habilitadas por Tecnologías de la Información y Comunicación (TIC) en Brasil, enfatizando las tecnologías utilizadas, los canales habilitados por las herramientas digitales, los procesos de aprendizaje para innovación y los cambios organizacionales necesarios para el uso de herramientas avanzadas. La metodología consiste en la adaptación de taxonomías existentes para los propósitos específicos de la investigación y la realización de un estudio de campo con la asistencia del software Atlas.ti. Los resultados muestran que las innovaciones habilitadas por TIC no constituyen eventos aislados y puntuales, ya que alteran procesos, formas de relacionarse con aliados y clientes, y requieren una mayor integración de funciones organizacionales. Fueron observadas importantes diferencias en función del tamaño de la empresa, de la internacionalización de las operaciones, de la capacitación técnica y del grado de acceso a herramientas propias.

PALABRAS CLAVE / Tecnologías de la Información y Comunicación, gestión, innovación, software, servicios. 


\section{INTRODUÇÃO}

O aumento do conteúdo informacional da economia mundial tem estimulado o desenvolvimento de empresas de software especializadas no provimento de serviços intensivos em conhecimento - Knowledge Intensive Business Services (KIBS) - responsáveis por suprir recursos críticos demandados pelas organizações dentro de uma lógica competitiva baseada em inovação. Empresas de software e serviços têm potencial de disseminar e habilitar inovações em todo o tecido produtivo, empregar pessoal qualificado e ser, ao mesmo tempo, principal produtor, comprador e usuário de TIC, contribuindo para aumentar a produtividade e a capacidade de inovação da economia como um todo (Chesbrough, 2011; Gallouj \& Savona, 2010; Miles \& Ducatel, 2005, Gago \& Rubalcaba, 2006, Miles, 2008; Nambisan, 2013).

A análise do uso de TIC nos processos de inovação em serviços enfrenta as dificuldades inerentes a produtos intangíveis, cuja produção é geralmente concomitante ao consumo e cujo efeito pervasivo é difícil de ser identificado e quantificado. Sabemos que ferramentas digitais sofisticadas oferecem condições para aperfeiçoar (e até revolucionar) processos de inovação e que determinadas firmas de serviço se encontram na vanguarda em termos de aplicação de TIC para inovação. Entretanto, existem muitas lacunas sobre os objetivos, instrumentos e características das inovações habilitadas por TIC, fato que motivou esta pesquisa sobre o uso de ferramentas computacionais no processo de inovação de empresas brasileiras de software e serviços de TI.

Três questões foram levantadas visando analisar o papel das TICs sobre o processo de inovação em empresas de serviços como um todo, abrindo caminho para pesquisas mais abrangentes em termos setoriais. A primeira refere-se ao que pode ser realizado, em termos de inovação, com a aplicação das ferramentas digitais disponíveis, especialmente na área organizacional. Dois pontos são investigados nessa questão: (i) Como as variáveis relativas ao porte e à origem do capital da empresa impactam o acesso a ferramentas para prospecção de informação, integração de redes e aprendizado? e (ii) Qual o papel das tecnologias free e/ ou open source na diversificação e especialização das atividades? A segunda questão refere-se às tecnologias, aos canais habilitados pelas ferramentas digitais e aos processos de aprendizado para inovação. 0 foco da análise reside em como as organizações conjugam diferentes ferramentas digitais para dar suporte às atividades de gestão, implementação e desenvolvimento de novos serviços e produtos. A terceira questão reside nas mudanças organizacionais necessárias para o uso de ferramentas mais avançadas de TIC. Qual o papel do porte da empresa e da cultura corporativa na implementação de inovações organizacionais?
A metodologia empregada envolveu duas etapas empíricas, executadas com base no método de análise de conteúdo qualitativo, com auxílio do software para tratamento de dados qualitativos Atlas.ti. A primeira fase consistiu na revisão da literatura especializada e consulta a pesquisadores acadêmicos, na Inglaterra e no Brasil, visando montar uma estrutura analítica e criar uma lista final de perguntas para aplicação às firmas-alvo da pesquisa. Isso permitiu desenvolver a segunda etapa, que buscou responder às questões da pesquisa por meio de entrevistas com empresas de software e serviços de TI no Brasil. Este artigo sintetiza a pesquisa realizada, enfatizando o tratamento teórico-conceitual da relação entre TIC e inovação, e as especificidades da inovação em serviços, para finalmente apresentar os resultados obtidos nos estudos de caso.

\section{TIC E INOVAÇÃO: EXPLORANDO UMA NOVA DIMENSÃO DE ANÁLISE}

A relação entre TIC e inovação permeia diferentes domínios do conhecimento e dimensões de análise. No campo econômico, a mensuração da chamada sociedade ou economia da informação é de particular interesse para órgãos oficiais de estatística que buscam entender os esforços e os impactos produzidos por essas tecnologias - notadamente na forma de equipamentos - sobre a produtividade (Antonelli, 2003; Black \& Lynch, 1997; Brynjolfsson \& Hitt, 2003; Greenan \& Mairesse, 1996; Lynch, 2004; Mendonça, Freitas, \& Souza, 2009; Paganetto, Becchetti, \& Bedoya, 2004), no nível e estrutura do emprego, salários e qualificações (Autor, Katz, \& Krueger, 1998; Falk, 2001; Giovanneti \& Menezes-Filho, 2003), e sobre a criação de vantagens competitivas sustentáveis (Carr, 2003).

Nos anos 1990, Robert Solow provocou os economistas com o que chamou de "paradoxo da produtividade", argumentando que se viam computadores em toda a parte, menos nos indicadores de produtividade. Referia-se aos resultados frustrantes da aplicação de TIC sobre o crescimento do valor adicionado por empregado, especialmente no setor serviços. Vários pesquisadores aceitaram o desafio de examinar o soi-disant paradoxo (Brynjolfsson 1993; Brynjolfsson \& Saunders, 2010), convergindo para a conclusão de que as medidas tradicionais de produtividade eram inadequadas, pelo menos em curto prazo, para medir os impactos econômicos das TIC. Para Brynjolfsson e Saunders (2010), uma medição apropriada dos insumos utilizados em cálculos de produtividade não pode ser limitada às horas trabalhadas, mas precisa incluir a qualidade dos equipamentos utilizados, a educação e treinamento da força de trabalho, e o capital organizacional. Graças a esses estudos, sabemos hoje que 
o principal impacto do uso de TIC nas organizações não reside propriamente na solução de gargalos ou na aceleração dos processos preexistentes, mas, sim, em inovações organizacionais mais radicais envolvendo a participação de novos agentes, ferramentas digitais, arranjos organizacionais, modelos de negócios e práticas gerenciais, que podem ser combinadas para gerar novos processos, bens e serviços. Uma significativa parcela do poder de impacto dessas tecnologias manifesta-se sobre variáveis qualitativas (Albertin \& Albertin, 2005, 2007; Licht \& Moch, 1999; Sanchez \& Albertin, 2009).

Com relação às teorias de inovação, duas perspectivas podem ser observadas. A primeira concentra-se nos efeitos dos investimentos em TIC, contemplando variáveis associadas à introdução de novos produtos e processos (Licht \& Moch, 1999; Paganetto et al., 2004). A segunda perspectiva estuda os impactos dessas tecnologias como inovações per se, ou seja, inovações nas TIC, entendidas como mudanças de processos baseadas em TIC (ICT innovations), podendo ser interpretadas fundamentalmente pelos movimentos de difusão e assimilação dessas tecnologias. Tal literatura oferece-nos um valioso apoio, à medida que se propõe a examinar os fatores que afetam a difusão e a assimilação de inovações dessa natureza. Porém, ela não é suficiente para analisar o uso de TIC como ferramenta orientada para integrar, intensificar e acelerar processos de inovação. 0 uso das TIC vem sendo estudado pela área de Gestão Organizacional, que tende a atribuir um papel coadjuvante à inovação, tratando-a como um dos vários benefícios que podem ser gerados pelas TIC, ao lado de outros elementos como custo, produtividade, qualidade e flexibilidade (Albertin \& Albertin, 2005, 2007).

Colocando a inovação no núcleo da investigação, esta seção procura tratar, conceitual e teoricamente, do potencial das TIC como suporte à inovação, enfatizando sua diversidade. A materialização disso, contudo, demanda tempo de experimentação e aprendizado, e envolve uma série de condicionantes relativos aos ambientes interno e externo às organizações, como veremos a seguir. Apesar das dificuldades em mensurar os impactos das TIC, entendemos que seu potencial como ferramentas de inovação tende a ser maior nos serviços do que na manufatura, dados seu caráter imaterial e a possibilidade de digitalização da maioria dos seus produtos.

\section{Ferramentas digitais de suporte à inovação: uma nova tipologia dentro de uma nova abordagem}

Um dos problemas mais frequentes enfrentados pelos autores que procuram classificar as TICs é a sistemática mudança nessas tecnologias ao longo do tempo. Isso levou Chen, Nunamaker, e
Weber (1989) a propor que as ferramentas sejam classificadas de acordo com suas funcionalidades básicas, as quais tendem a permanecer estáveis por mais tempo. Seguindo essa linha, Dodgson, Gann, e Salter (2005) caracterizam a inovação contemporânea como um processo dinâmico representado pela interação de três atividades simultâneas e assistidas por ferramentas digitais: (i) pensar (think), (ii) experimentar (play) e (iii) implementar ou produzir (do).

Com base no esquema Think, Play, Do, os autores sugerem uma tipologia genérica que classifica as tecnologias digitais em três grupos, segundo o papel predominante que desempenham no processo de inovação (Figura 1): (i) Tecnologias de Informação e Comunicação (TIC); (ii) Tecnologias de Operação e Manufatura (TOM); e (iii) Tecnologias de Inovação (TIV).

\section{Figura 1. Integração entre ferramentas tecnológicas genéricas}

\begin{tabular}{|c|c|c|}
\hline 7 & & $\begin{array}{l}\text { Tecnologias de Inovação } \\
\text { Ferramentas de busca } \\
\text { Modelagem e simulação } \\
\text { Visualização/Realidade Virtual } \\
\text { Prototipagem rápida }\end{array}$ \\
\hline Tecnologia de & Tecnologias de & Tecnologias de Design e \\
\hline Informação: & Comunicação: & Produção: \\
\hline $\begin{array}{l}\text { Computadores } \\
\text { e serviços }\end{array}$ & $\begin{array}{l}\text { Internet/www } \\
\mathrm{WiFi} / 3 \mathrm{G}\end{array}$ & CINS, FMS, CAD, CAM, CIM, CIP \\
\hline $\begin{array}{l}\text { Sistemas } \\
\text { abertos }\end{array}$ & $\begin{array}{l}\text { Troca eletrônica } \\
\text { de Dados (EDI) }\end{array}$ & $\begin{array}{l}\text { Tecnologias de Networking e } \\
\text { Coordenação: }\end{array}$ \\
\hline $\begin{array}{l}\text { Banda larga } \\
\text { Sensores }\end{array}$ & & MRP, ERP, PMS, TQM, JIT \\
\hline
\end{tabular}

O esquema Think, Play, Do coloca a inovação sob um prisma diferente do tradicional, permitindo incorporar características mais recentes do processo inovativo, a exemplo da emergência dos KIBS. Seus criadores ressalvam que o esquema não se aplica apenas a setores novos e emergentes, mas também àqueles tradicionais (manufatureiros), assim como aos setores público e de serviços.

\section{Revisitando o esquema Think, Play, Do}

No intuito de aperfeiçoar a adequação do esquema de Dodgson et al. (2005) à dinâmica de inovação em serviços, introduzimos modificações que partem de dois questionamentos principais. Primeiro, se o software pode integrar tecnologias tanto do lado 
da informação quanto do lado da comunicação, não parece mais sensato considerar TIV e TOM também como TIC? Segundo, ao analisar as ferramentas que compõem o conjunto do que Dodgson et al. (2005) chamam de TIV (Figura 1), podemos concluir que os autores estariam privilegiando aquelas ferramentas mais diretamente associadas ao desenvolvimento de novos produtos. Isso implica colocar em um plano secundário inovações de conteúdo não tecnológico (como mudanças de gestão ou organizacionais).

Visando incorporar esses pontos na análise do processo de inovação, nossa proposta encaminha-se no sentido de considerar todas as aplicações de software como TIC e ampliar o escopo daquilo que se pode considerar como inovação, atribuindo igual relevância às mudanças de natureza tecnológica e aquelas de caráter organizacional e de gestão. Como resultado, teríamos a seguinte tipologia:

(I) TICdi: voltadas prioritariamente ao suporte de atividades de desenvolvimento de inovações em bens e serviços, compreendendo fundamentalmente as tecnologias de design e prototipagem (virtual, no caso de software).

(II) TICii: dirigidas principalmente ao apoio às atividades de implementação ou operacionalização de inovações.

(III) TICgi: aplicadas predominantemente na assistência às atividades de gestão da inovação, incluindo tecnologias de integração de sistemas e redes e de gerenciamento mais amplo (não se restringindo a P\&D) do processo de inovação.

(IV) TICie: representam as TICs de infraestrutura digital.

No esquema revisitado, julgamos mais conveniente deslocar algumas aplicações de software, que na Figura 1 haviam sido categorizadas como TIC, para as tecnologias de gestão (TICgi), que, a exemplo dos sistemas Enterprise Resource Planning (ERP) e Customer Relationship Management (CRM), apoiam atividades de gestão do processo de inovação.

Considerando que a TICie já vem sendo alvo da grande maioria dos estudos consultados sobre TIC, assim como dos levantamentos estatísticos oficiais, o foco da nossa análise é dirigido para as demais categorias, ou seja, TIC de desenvolvimento, implementação e gestão da inovação. Seguindo a mesma lógica utilizada para reclassificar as tecnologias em consonância com as funções principais e preponderantes desempenhadas no suporte à inovação, é necessário também reclassificar as próprias atividades de inovação, as quais, em substituição a think, play e do, passariam a se chamar gestão, desenvolvimento e implementação da inovação.

A Figura 2 ilustra o modelo Think, Play, Do revisitado, o qual tem seu nome alterado para Modelo de Gestão, Desenvolvimento e Implementação da Inovação Auxiliados por TIC (GDI-TIC), onde a integração entre tecnologias está representada pela interseção entre as quatro elipses no plano inferior, e TICie aparece como a infraestrutura digital (hardware e software) sobre a qual operam as outras três categorias de ferramentas digitais (aplicações de software para computador) em tese mais diretamente envolvidas com o processo de inovação.

\section{Figura 2. 0 modelo GDI_TIC}

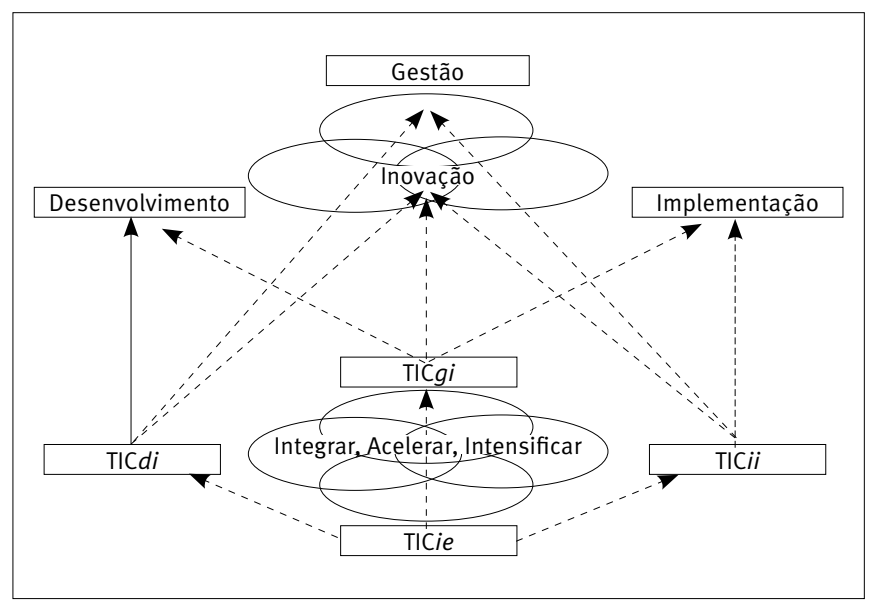

Essa configuração proporciona condições potenciais para integrar, acelerar e intensificar o processo de inovação, o qual se encontra simbolizado pela interação entre as três elipses no plano superior referentes às atividades de gestão, desenvolvimento e implementação. As setas que partem das tecnologias ( $\mathrm{gi}$, di e ii) em direção ao processo de inovação representam os canais por meio dos quais o potencial dessas tecnologias pode se materializar, ou seja, as formas como essas aplicações de software para computador auxiliam o processo de inovação.

Assumimos que TICdi e TICii têm como função predominante, respectivamente, o suporte direto às atividades de desenvolvimento e implementação (setas cheias). As TICgi, por seu turno, oferecem, a princípio, suporte a todas as atividades, direta ou indiretamente (setas pontilhadas), em especial às de gestão da inovação (agora também com seu âmbito ampliado, incluindo as atividades think).

A primazia de inovações tecnológicas na análise do esquema Think, Play, Do original sugere um escopo mais limitado para a atividade think. No esquema revisitado, a atividade de gestão da inovação possui uma dimensão maior, de maneira a incorporar atividades mais amplas de gestão nas firmas (e, 
consequentemente, oferecer suporte ao surgimento de inovações organizacionais).

A mesma ideia aplica-se às atividades de desenvolvimento, que passam a incluir - e privilegiar - atividades não formalizadas de Pesquisa e Desenvolvimento (P\&D) - com foco no “D” - mais próximas do processo de inovação das firmas de serviços e, por esse motivo, mais propícias a receber suporte de TICdi. Essas modificações parecem-nos mais compatíveis com a dinâmica dos serviços e dos modernos processos de inovação.

Uma vez que procuramos aplicar uma abordagem para a relação entre TIC e inovação em firmas de serviço, fugindo dos approaches tradicionais, é preciso, a partir de então, ponderar especificidades da inovação em serviços (como um produto) e da dinâmica inovativa das firmas desse setor (como processo), em particular o de serviços de TI.

\section{INOVAÇÃO E SERVIÇOS}

\section{Características dos serviços}

Entre as tentativas de explicar inovação em serviços, encontramos em Miles (2008) uma interessante e atualizada linha de argumentação, inspirada em trabalhos anteriores (Green, Miles, \& Ruter, 2007; Hertog, 2000; Toinoven \& Tuominen, 2007) e fundamentada na ideia de que determinadas características - gerais e intrínsecas - dos serviços, juntamente com certos fatores que os diferenciam, respondem em boa medida pelos padrões setoriais de inovação observados.

Algumas características são comuns a praticamente todos os serviços; outras são mais específicas. Para os nossos propósitos, voltaremos atenção primordialmente àquelas que ajudam a definir os contornos principais da inovação nos KIBS e nos Technology-Intensive KIBS (T-KIBS), onde se incluem os serviços de TI. Para Abreu, Grinevich, Kitson, e Savona (2010), inovações em KIBS geram cinco vezes mais valor adicionado para a economia da Grã-Bretanha do que na manufatura.

Um produto-serviço constitui, segundo Miles (2008), um conjunto de funções, que representam os principais produtos fornecidos por determinada indústria de serviço. Ao se produzir um serviço, o foco principal não é fornecer um bem (material), mas organizar uma solução para um problema, colocando à disposição de um cliente uma cesta de capacitações e competências (Hertog, 2000).

Essa exposição abre espaço para a diferenciação entre produtos de serviço altamente padronizados, com características próximas a um bem físico (exemplo: cadeias de fast-food), e serviços mais customizados, muitas vezes baseados em formas mais tácitas de conhecimento. Nesse último caso, os serviços - não raro - surgem como um resultado da coprodução, mobilizando provedor e cliente, como é o caso, por exemplo, dos serviços de consultoria. Tais definições ajudam a identificar duas características comuns compartilhadas pelos serviços:

(I) Intangibilidade - implica que os produtos de serviço (em contraposição aos materiais) tipicamente envolvem transformações no estado de determinadas entidades, que podem ser artefatos (produtos materiais), pessoas (ou outros organismos, como animais) ou dados (símbolos).

(II) Interatividade - reflete a necessidade de presença e participação do cliente em muitos processos de serviços, onde há casos compreendendo seu envolvimento ativo e conjunto com a empresa provedora, como no design e/ou na produção de certos serviços de TI (Djellal \& Gallouj, 1999; Hipp \& Grupp, 2005; Miles \& Ducatel, 2005, Miles, 2008).

Miles (2008) sugere que intangibilidade e interatividade estão, muitas vezes, associadas a três outras características típicas de muitos serviços: (iii) simultaneidade - produto, processo e consumo ocorrem ao mesmo tempo e no mesmo espaço; (iv) baixa portabilidade - dificuldade em armazenar e transportar produtos de serviço; e (v) intensidade de informação - assinala a importância dos fluxos de comunicação entre os agentes.

Outra questão, com importantes desdobramentos para a inovação, reporta-se às três formas principais por meio das quais os serviços diferem entre si:

(I) Processos fundamentais ou transformações: fazem alusão à relação na qual o tipo de transformação operada pelos serviços e os efeitos gerados sobre determinado objeto estabelece a modalidade de conhecimento requerido nas operações de serviço e os novos conhecimentos necessários para inovação.

(II) Intensidade de conhecimento: faz referência ao nível de qualificação exigido por determinada atividade de serviço daqueles trabalhadores que exercitam habilidades profissionais ou técnicas para gerar um resultado específico a uma determinada situação.

(III) Relações de mercado: associa o processo de inovação com a extensão em que os serviços atendem (ou são financiados por) consumidores, empresas privadas ou 
setor público e o grau em que o serviço é altamente customizado, em contraposição ao produto padronizado e de produção em massa.

No que tange aos três fatores acima, a ideia central é que diferentes atividades de transformação envolvem distintos recursos de equipamentos e materiais, assim como a aplicação de diferentes habilidades, bases de conhecimento, profissões e comunidades de usuários que influenciarão a estrutura e condução da inovação. A identificação do tipo de transformação baseia-se no tipo de objeto a ser transformado. Os T-KIBS poderiam, assim, ser incluídos entre os serviços intensivos em informação, uma vez que se concentram no consumo (ou na transformação) desse recurso, o que, do ponto de vista da inovação, aponta para uma trajetória fortemente baseada no uso de novas TICs. O segundo elemento diferenciador (intensidade de conhecimento) refere-se aos serviços como processadores de informação e conhecimento nos quais o nível educacional dos trabalhadores é usualmente utilizado como proxy de conhecimento requerido. A maior presença relativa de trabalhadores altamente qualificados nos KIBS sugere a aplicação de conhecimento especializado para realizar transformações no processo de desenvolvimento e implementação de software. Isso pode ser relacionado ao uso frequente e intenso de TIC, incorporando maior grau de complexidade na resolução de problemas. 0 terceiro componente diferenciador (relações de mercado) faz referência aos serviços como provedores de soluções para clientes específicos.

Definidas as principais características e fatores diferenciadores dos serviços, o próximo passo é criar uma conexão entre esses componentes e padrões de inovação. Trata-se de uma tarefa complexa, devido à enorme heterogeneidade existente nos serviços.

\section{Padrões de inovação}

Baseando-se no entendimento de que a estrutura conceitual do Manual de Inovação da The Organisation for Economic Co-operation and Development (OECD, 2005) ainda está atrelada à era industrial, vários pesquisadores de inovação em serviços defendem sua reformulação para melhor lidar com as características dos novos processos de inovação e procedimentos de mensuração estatística em serviços (Djellal \& Gallouj, 1999; Green et al., 2007; Hertog, 2000; Hipp \& Grupp, 2005; Miles \& Ducatel, 2005, Miles, 2008).

Algumas premissas relativas aos serviços normalmente são assumidas por esses autores: uma delas aponta que intangibilidade, interatividade e simultaneidade confluem para dificultar, em muitas situações, a diferenciação entre inovação de produto e de processo. Adicionalmente, o modo predominantemente não formal de organização das atividades, assim como a incidência de inovações introduzidas pelo próprio usuário (Oliveira \& Hippel, 2011), influencia a prevalência de processos contínuos de inovação, que, por sua vez, limitam a emergência de inovações radicais.

Miles (2008) acrescenta que os serviços normalmente apresentam uma proporção relativamente maior de empresas pequenas, produzindo efeitos sobre a organização da inovação. Grandes empresas são mais propensas a desenvolver inovações no âmbito de estruturas organizadas e formais, envolvendo grupos funcionais especializados. A inovação de serviço, por seu turno, raramente se restringe às características do produto em si, dado que coincide com novas formas de distribuição do produto, de interface com o cliente, de controle de qualidade e segurança.

Essas premissas, embora relevantes, remetem a um quadro bastante geral dos serviços. Para descer a níveis mais específicos, precisamos de instrumentos analíticos adicionais, que nos ajudem a olhar setorialmente para características da inovação de serviço e da dinâmica de inovação em empresas desse setor.

\section{Faces da inovação}

Um ponto de partida útil é identificar a inovação em serviços como um fenômeno compreendendo três faces amplas: (i) tipos, (ii) gerenciamento e (iii) contexto (ou organização mais ampla) da inovação (Green et al., 2007; Miles, 2008).

\section{(I) Tipos de inovação}

Representam o foco da novidade que, segundo Hertog (2000), estão relacionados a quatro dimensões: conceito de serviço, interface com o cliente, sistema de distribuição e opções tecnológicas.

a. Conceito de serviço: a inovação pode envolver uma ideia para o desenvolvimento de um serviço, ou seja, uma nova proposição de valor, que seja nova pelo menos para a própria firma. Embora nem todas as inovações de serviço apresentem um forte componente novo, inovações conceituais são mais marcantes nessas firmas do que naquelas puramente manufatureiras. As inovações de serviço são, em geral, intangíveis, significando que, em alguns casos, novas características possuem pouca relação com artefatos materiais, não obstante o serviço em si ter elementos bastante tangíveis (exemplo: terminais bancários eletrônicos). 
b. Interface com o cliente: refere-se a mudanças na maneira como os clientes são envolvidos no design, produção e consumo do serviço.

c. Sistema de distribuição do serviço: esta dimensão muitas vezes relaciona-se diretamente à anterior, uma vez que a distribuição é uma das modalidades específicas de interação, que ocorre por meio da interface com o cliente. As inovações, neste caso, dizem respeito a mudanças internas nas formas pelas quais trabalhadores executam a distribuição de serviços. A introdução do comércio eletrônico em processos de negócios (business processes) pode ser um exemplo deste tipo de inovação.

d. Opções tecnológicas: equivalem às inovações de processo do Manual de Inovação da OECD, bastante familiares na manufatura. 0 primeiro uso de ferramentas em ambiente CASE na criação de software pode ilustrar uma inovação desta natureza.

Na realidade, as conexões entre as dimensões podem ser até mais importantes do que elas isoladamente, e, na prática, a sua combinação é que caracteriza uma inovação de serviço particular.

Questões relativas à coordenação e integração do processo de inovação têm a ver com as outras duas faces do modelo de Miles (2008): o gerenciamento e o contexto ou organização mais ampla da inovação.

(II) Gerenciamento da inovação

Corresponderia ao processo, no nível da firma, por meio do qual novas ideias são geradas, selecionadas e materializadas dentro de novas práticas e produtos, que podem, então, ser testados, difundidos, implementados e configurados. Explorando a inovação em KIBS, Toinoven e Tuominen (2007) diferenciam cinco processos, cuja variação repousa na extensão em que há formalização do processo de inovação e nas modalidades de colaboração buscadas: a) Processos internos sem um projeto específico; b) Projetos internos de inovação; c) Projetos de inovação com um cliente-piloto; d) Projetos de inovação customizados para um cliente; e e) Projetos de inovação com financiamento externo.

Essas cinco modalidades refletem a multiplicidade de práticas de inovação em firmas de serviço. Além disso, as autoras chamam atenção para o caráter dinâmico desses processos, uma vez que, ao longo do seu ciclo, uma inovação iniciada sem um esforço consciente pode assumir a forma de procedimentos mais sistemáticos.
(III) Contexto geral ou organização mais ampla da inovação

A terceira face da inovação remete ao seu contexto sistêmico, compreendendo não apenas a empresa mas também os fluxos de ideias e artefatos entre organizações e redes; e mesmo instituições fora do sistema (exemplo: ministérios lidando com taxação/créditos fiscais, políticas de emprego e capacitação) podem afetar o processo de inovação. Nesse caso, é correto pensar em termos de sistema nacional, regional e setorial de inovação, conexões das firmas à jusante e à montante, assim como a noção de cluster e de cadeia de valor, com chance de, inclusive, assumir um caráter global (Green et al., 2007; Miles, 2008).

\section{Interação entre as faces da inovação}

As três faces da inovação (tipos, gerenciamento e contexto geral) costumam estar relacionadas entre si e com uma quarta categoria: o agente inovador. A natureza da organização inovadora tende a influenciar e ser influenciada pelo tipo de inovação realizada, pela forma como é gerenciada e pelo contexto(s) sistêmico(s) onde o processo de inovação se inscreve (Figura 3).

\section{Figura 3. Interação entre as faces da inovação}

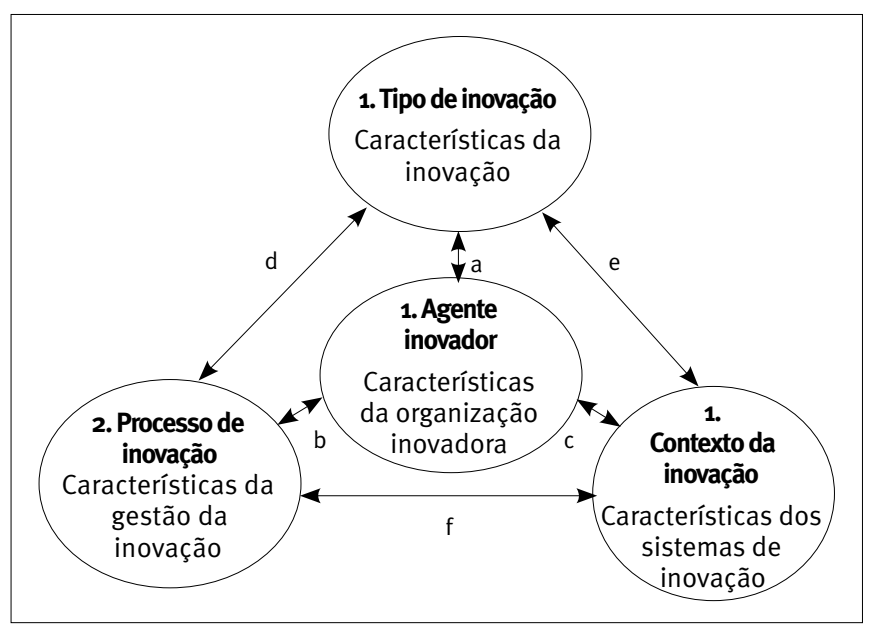

Inúmeras possibilidades de interação emergem do caráter dinâmico, sistêmico e complexo do processo de inovação. A Figura 3 ilustra possíveis direções gerais (e potenciais) de causalidade recíproca entre as faces da inovação (identificadas como a, b, c, d, e, f), as quais são descritas por Green et al. (2007) e sintetizadas a seguir.

a. Firmas em setores particulares tendem a realizar tipos específicos de inovação, refletindo produtos e processos utilizados na sua produção e distribuição; 
empresas de portes distintos terão acesso a diferentes recursos para investir em inovação, de novo influenciando as inovações buscadas.

b. Organizações são propensas a herdar formas setoriais específicas de gerenciamento da inovação em função do setor de atividades em que estão inseridas, do tamanho e de sua especialização na divisão internacional do trabalho.

c. Empresas dentro de setores determinados normalmente possuem acesso a recursos para inovação. Grandes empresas podem ter acesso a redes mais amplas e mais recursos para investir na busca por informação e colaboração.

d. É provável que o tipo de inovação realizada crie demandas específicas por gerenciamento da inovação, dependendo das formas de conhecimento e profissão mobilizadas, do tipo de mercado e de financiamento buscados.

e. Alguns tipos de inovação são representativos, na condição de frutos de iniciativas tomadas por determinados atores como conselhos de pesquisa (para desenvolver conhecimento relevante) e intermediários de negócios (para difundir tecnologias importantes); outros são menos articulados.

f. O gerenciamento da inovação por parte de uma organização pode afetar sua relação com o contexto mais amplo da inovação (por exemplo, uma firma instigando processos de inovação aberta será mais propícia a fluxos de conhecimento com colaboradores, fornecedores e clientes), ao passo que o contexto pode moldar as práticas de gerenciamento (Bueno \& Balestrin, 2012).

Devemos reconhecer a importância de modelos, uma vez que nos oferecem lentes para analisar um determinado fenômeno. Não obstante, eles - na condição de simplificadores de uma determinada realidade - podem apresentar limitações, sobretudo quando aplicados a contextos específicos e setores de atividade econômica dentro dos quais há diversidade respeitante a elementos como porte de firmas, origem do capital (nacional ou estrangeira), condições de mercado, divisão internacional do trabalho, trajetórias e ambiente institucional.

\section{USO DE TIC PARA INOVAÇÃO EM FIRMAS DE SERVIÇOS DE TI}

Apresentamos a seguir uma análise comparativa dos estudos de caso realizados em nossa pesquisa sobre o uso de software como ferramenta para inovação em empresas brasileiras de serviços de $\mathrm{TI}$, ponderando especificidades da inovação em serviços apresentadas anteriormente.

\section{Procedimentos metodológicos}

À luz do que foi apresentado, iniciamos criando uma lista preliminar de perguntas sobre o tema, inspirada no modelo de Gestão, Desenvolvimento e Implementação da Inovação Auxiliados por TIC (GDI-TIC), um modelo conceitual proposto pelos autores a partir de seu background teórico, revisitando o esquema Think, Play, Do. Essa lista foi submetida, entre 2009 e 2010 , a seis pesquisadores acadêmicos na Inglaterra (do Manchester Institute of Innovation Research e do London Imperial College) e a dois no Brasil (do Instituto de Economia da UFRJ), primeira fase empírica, buscando seu aperfeiçoamento e validação. Tanto essas consultas quanto os preceitos subjacentes ao modelo GDI_TIC e às teorias sobre inovação em serviços fundamentaram a elaboração de uma estrutura analítica geral, que instrumentalizou a confecção da lista final de questões, utilizada em entrevistas presenciais conduzidas em 2010 com gestores de firmas da IBSS (segunda fase empírica).

O tratamento do material das entrevistas, armazenadas em aparelho gravador de voz, seguiu o método de análise de conteúdo qualitativo com auxílio do software de Atlas.ti, que permite trabalhar em dois níveis fundamentais: o textual, incluindo atividades como segmentação de arquivos de dados, codificação de texto e criação de memos para formação e estruturação de teorias; e o nível conceitual, mais centrado em atividades de construção e/ou estruturação de modelos, a exemplo da conexão de códigos a networks.

Na linguagem do Atlas.ti, cada uma de nossas fases empíricas corresponde a uma Unidade Hermenêutica ou Projeto; e cada entrevista equivale a um Documento Principal, contendo como elementos essenciais códigos ou categorizações, marcações de texto, memos e comentários. A ferramenta permite que estes e outros componentes possam ser conectados entre si em networks com diversas triangulações e representações visuais (network views), obedecendo a relações hierarquizadas (diferentes níveis conceituais de abstração) e teóricas (causalidades simétricas ou assimétricas, contraditórias, transitivas etc.). 
A Figura 4 apresenta uma visão de rede, recurso visual disponibilizado pelo software, que sintetiza a estrutura analítica, as categorias-chave de nossa pesquisa e as principais hierarquias conceituais e direções de causalidade.

Na parte inferior, encontram-se os três tipos de tecnologias analisadas: TIC de suporte ao desenvolvimento de inovações (TICdi), à gestão da inovação (TICgi) e à implementação de inovações (TICii). Essas tecnologias habilitam canais (exemplo: integração interna e externa da empresa, produtividade e coordenação de processos), por meio dos quais inovações de serviço (de conceito, interface com o cliente, sistema de distribuição e opções tecnológicas) e mudanças organizacionais (em termos gerenciais, nas estratégias de negócios e de marketing) podem surgir. Contudo, isso depende de processos de aprendizado e está sujeito a barreiras inerentes aos ambientes interno e externo à empresa. O Quadro 1 descreve as categorias analíticas da Figura 4.

\section{Figura 4. Estrutura analítica geral: visão de rede (network view) das principais categorias}

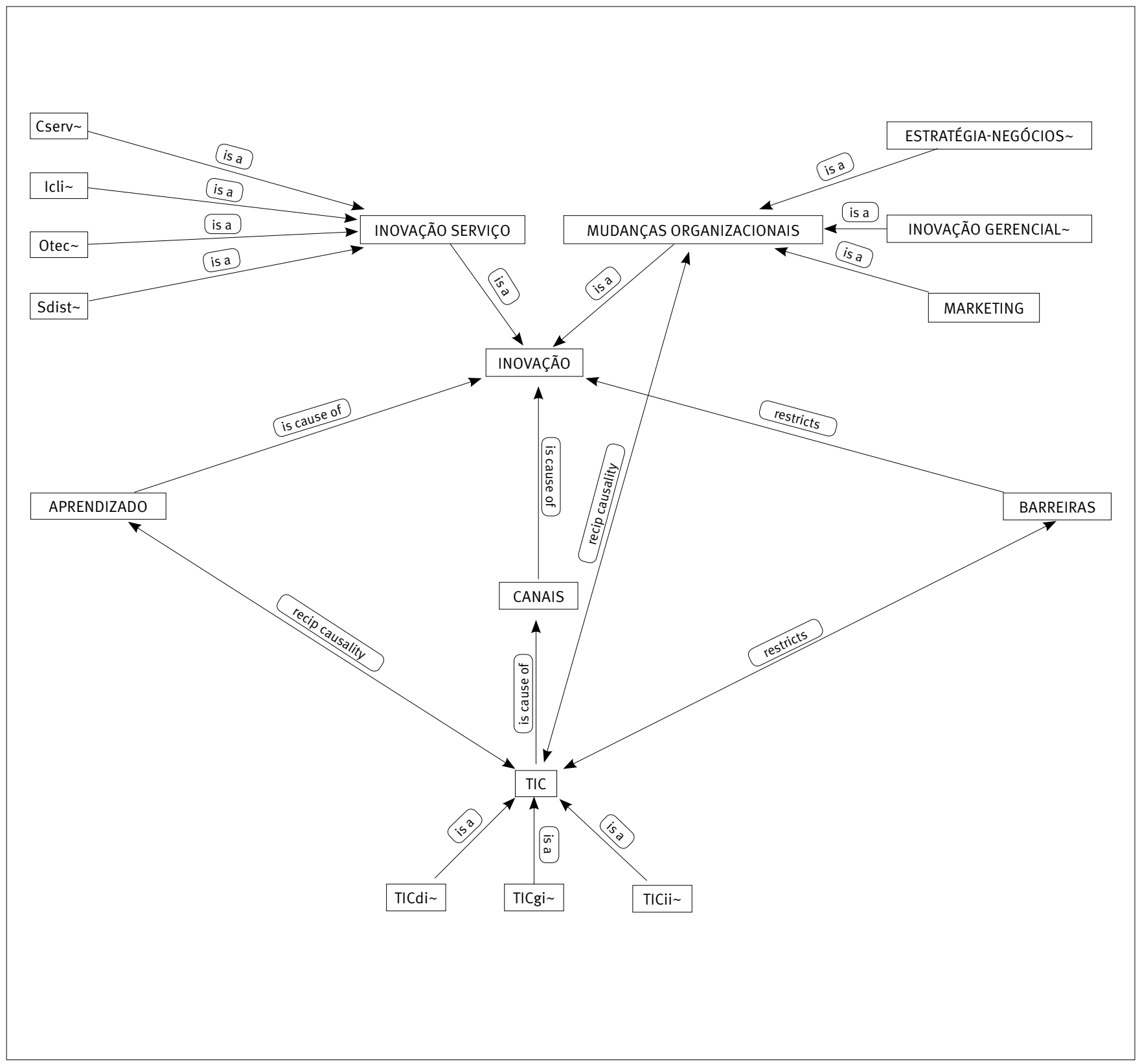

Fonte: Recuperado da ferramenta Atlas.ti.

Notas: Na terminologia do Atlas.ti, "is a” significa "constituir uma espécie de determinada categoria”; “is cause of” significa "causar”; “recip causality” significa "causalidade recíproca”; "restricts” corresponde "a impor restrições”. 
Quadro 1. Estrutura analítica geral: descrição das categorias-chave

Modelo de Gestão, desenvolvimento e implementação da inovação auxiliados por TIC (GDI_TIC)

\begin{tabular}{l|l|l}
\hline Atividades de inovação & TIC de suporte & Exemplos de funções \\
\hline Gestão & TIC gi & $\begin{array}{l}\text { Integração de sistemas e redes } \\
\text { gerenciamento ou coordenação }\end{array}$ \\
\hline Desenvolvimento & & $\begin{array}{l}\text { Modelagem } \\
\text { Simulação } \\
\text { Otimização } \\
\text { Visualização } \\
\text { Prototipagem } \\
\text { Prospeção de informação }\end{array}$ \\
\hline Implementação & TIC di & Opracionalização \\
\hline
\end{tabular}

\section{Faces da inovação e serviços}

Faces da inovação

Especificações de mudança na firma

Inovação de serviço - tipos

(dimensões)
Conceito de serviço (Cserv)

Interface com o cliente (Icli)

Sistemas de distribuição (Sdist)

Opções tecnológicas (Otec)

Mudanças organizacionais ou de gestão
Estratégias-áreas de negócio

the

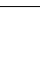

Marketing

Características dos sistemas de inovação
Grau de formalização

Técnicas gerenciais

Modos de organização do trabalho

Diversificação

Especialização

Outsourcing

Off-shoring

Técnicas de promoção

Canais de venda

Métodos de fixação de preços

Baseando-se nessa estrutura, uma lista de temáticas e questões (Quadro 2) foi utilizada em entrevistas com gestores de sete firmas de serviços de TI localizadas no Rio de Janeiro. A seleção das firmas orientou-se no sentido de capturar algo da heterogeneidade que marca o setor de serviços de TI, incluindo empresas de serviços de software (i) de alto e baixo valor adicionado; (ii) grandes, médias, pequenas e microempresas; e (iii) de capital de origem nacional e estrangeira. 
Quadro 2. Lista final de áreas temáticas e questões apresentadas a gestores privados da IBSS

\begin{tabular}{|c|c|c|}
\hline Áreas Temáticas & Questão & Formulação da questão \\
\hline \multirow{4}{*}{$\begin{array}{l}\text { (i) Aplicação de TIC para } \\
\text { Inovação }\end{array}$} & 1 & $\begin{array}{l}\text { A utilização de Aplicações de Software (AS) pode contribuir significativamente para que empresas } \\
\text { criem coisas novas (inovações). Isso ocorreu com as principais mudanças implementadas pela sua } \\
\text { empresa nos últimos anos? Caso sim, responda a q. 2. Caso não, passe para a q. } 10 .\end{array}$ \\
\hline & 2 & $\begin{array}{l}\text { Quais os tipos principais de coisas novas que sua empresa conseguiu introduzir nos últimos anos } \\
\text { com o suporte de AS? }\end{array}$ \\
\hline & 3 & $\begin{array}{l}\text { Em que funções/atividades principais sua empresa utilizou AS no processo de inovação nos últimos } \\
\text { anos? Especifique os principais tipos de AS utilizadas na execução dessas funções e informe quais } \\
\text { dessas AS foram desenvolvidas por sua empresa, isoladamente ou em parceria. }\end{array}$ \\
\hline & 4 & $\begin{array}{l}\text { De que forma as AS mencionadas por você ajudaram no processo de inovação da sua empresa nos } \\
\text { últimos anos? }\end{array}$ \\
\hline $\begin{array}{l}\text { (ii) Processos de } \\
\text { aprendizado }\end{array}$ & 5 & $\begin{array}{l}\text { Como os funcionários de sua empresa aprenderam a utilizar AS no processo de inovação nos últimos } \\
\text { anos? }\end{array}$ \\
\hline \multirow{2}{*}{$\begin{array}{l}\text { (iii) Mudanças } \\
\text { Organizacionais dando } \\
\text { Suporte ao uso de } \\
\text { AS no processo de } \\
\text { Inovação }\end{array}$} & 8 & Essas mudanças ocorreram antes ou após o início da utilização de AS visando a inovação? \\
\hline & 9 & Qual foi o principal papel dessas mudanças para auxiliar o uso de AS no processo de inovação? \\
\hline $\begin{array}{l}\text { (iv) Barreiras ao uso de } \\
\text { TIC para inovação }\end{array}$ & 10 & $\begin{array}{l}\text { Quais as principais dificuldades enfrentadas pela sua empresa para iniciar o uso ou durante o } \\
\text { processo de utilização de AS visando a inovação nos últimos anos? Considere barreiras relativas aos } \\
\text { ambientes interno e externo à sua empresa. }\end{array}$ \\
\hline
\end{tabular}

Solicitamos a cada entrevistado indicações de empresas com as quais sua organização mantinha relações de negócios (clientes e/ou fornecedores), de modo a observar interações entre atores nos sistemas de inovação. 0 Quadro 3 mostra o painel de empresas e informações associadas, obedecendo à ordem cronológica de condução das entrevistas. Na sequência, analisamos os resultados das entrevistas com gestores de firmas de serviços de TI.

\section{Quadro 3. Painel de empresas entrevistadas nos estudos de caso}

\begin{tabular}{|c|c|c|c|c|c|c|}
\hline Empresas & $\begin{array}{l}\text { Origem do } \\
\text { capital }\end{array}$ & Sede & $\begin{array}{l}\text { N. funcionários no } \\
\text { Brasil }^{\star \star} / \\
\text { Tamanho } \\
\end{array}$ & $\begin{array}{l}\text { Nicho } \\
\text { principal }\end{array}$ & $\begin{array}{l}\text { Nome do Entrevistado/ } \\
\text { Cargo }\end{array}$ & $\begin{array}{l}\text { Duração da } \\
\text { entrevista }\end{array}$ \\
\hline $\begin{array}{l}\text { K\&M Serviços de } \\
\text { Manutenção Ltda. }\end{array}$ & Nacional & RJ & 50 (porte médio) & $\begin{array}{l}\text { Serviços de } \\
\text { baixo VA }\end{array}$ & $\begin{array}{l}\text { Felipe Deco (gerente } \\
\text { de TI) }\end{array}$ & 45 minutos \\
\hline Marlin Soluções Web & Nacional & RJ & 61 (porte médio) & $\begin{array}{l}\text { Serviços de } \\
\text { alto VA }\end{array}$ & $\begin{array}{l}\text { Gianini Saciloto (sócio } \\
\text { diretor de operações } \\
\text { de TI) }\end{array}$ & $\begin{array}{l}1 \text { hora e } 13 \\
\text { minutos }\end{array}$ \\
\hline FPS Informática Ltda. & Nacional & RJ & 8 (porte micro) & $\begin{array}{l}\text { Serviços de } \\
\text { baixo VA }\end{array}$ & $\begin{array}{l}\text { Fernando Pinho (diretor } \\
\text { executivo) }\end{array}$ & 57 minutos \\
\hline Alpha do Brasil* & Estrangeiro & n.d. & 7.000 (porte grande) & $\begin{array}{l}\text { Serviços de } \\
\text { alto VA }\end{array}$ & $\begin{array}{l}\text { George William* } \\
\text { (consultor de } \mathrm{TI} \text { ) }\end{array}$ & 42 minutos \\
\hline Wage Mobile & Nacional & RJ & 5 (porte micro) & $\begin{array}{l}\text { Serviços de } \\
\text { baixo VA }\end{array}$ & $\begin{array}{l}\text { Luiz Silveira (sócio } \\
\text { diretor de TI) }\end{array}$ & 37 minutos \\
\hline Unisys Brasil Ltda. & Estrangeiro & $\begin{array}{l}\text { Chicago/ } \\
\text { EUA }\end{array}$ & 3.000 (porte grande) & $\begin{array}{l}\text { Serviços de } \\
\text { alto VA }\end{array}$ & $\begin{array}{l}\text { Jandira Cristina } \\
\text { (gerente de projetos) }\end{array}$ & $\begin{array}{l}1 \text { hora e } 10 \\
\text { minutos }\end{array}$ \\
\hline $\begin{array}{l}\text { Clavis Segurança da } \\
\text { Informação }\end{array}$ & Nacional & $\mathrm{RJ}$ & 22 (porte pequeno) & $\begin{array}{l}\text { Serviços de } \\
\text { baixo VA }\end{array}$ & $\begin{array}{l}\text { Bruno Salgado (diretor } \\
\text { executivo) }\end{array}$ & 55 minutos \\
\hline
\end{tabular}

Notas: * Nomes fictícios.

** Número de funcionários existentes na data da entrevista.

$\mathrm{RJ}=$ Rio de Janeiro; n.d. = não disponível; $\mathrm{VA}=$ Valor Adicionado. 


\section{Análise geral e comparativa das entrevistas}

A análise privilegia a observação dos recortes mencionados (nicho de mercado, porte e origem do capital das firmas), sendo igualmente apresentada em conformidade com as áreas temáticas abordadas.

\section{(I) Aplicação de TIC para inovação}

Os estudos de caso mostram que as dimensões de uma inovação de serviço não são vetores discretos de mudança, uma vez que, em diversas situações, foram conjugados novos conceitos, formas de interface com o cliente, tecnologias e processos de distribuição, e isso foi, em grande parte, motivado pela integração de funções e, consequentemente, de atividades de inovação habilitada por determinadas TICs, sobretudo as mais sofisticadas.

É importante ter em mente o fato de que, embora tratemos de serviços, lidamos concomitantemente com um setor altamente intensivo em tecnologia (T-KIBS), o que parece explicar a incidência e relevância, em todas as empresas, de inovações nas opções tecnológicas. Essa orientação tecnológica, a exemplo do que ocorre tipicamente nas manufaturas, sugere uma dinâmica na qual o início de uso de uma nova tecnologia possui certa primazia no processo de inovação, desencadeando mudanças nas outras dimensões da inovação de serviço e de natureza organizacional.

Empresas atuantes em nichos de VA relativamente mais elevado tendem a diferenciar-se das demais pela capacidade de utilizar TIC no suporte às três atividades de inovação (gestão, desenvolvimento e implementação), por meio da potencialização de suas capacitações e habilitação de canais direcionados a mudanças organizacionais e de serviço.

A empresa Marlin Soluções Web, a despeito de ser nacional e de médio porte, pode ser destacada em termos de esforços inovativos. Isso pode ser observado pelo número de tecnologias utilizadas, pelas inovações introduzidas e pelo grau de sofisticação, qualidade e diversidade dos canais habilitados pelas tecnologias. Observam-se também interações mais robustas (inclusive, em atividades de desenvolvimento) com clientes, fornecedores e universidades e mudanças de gestão, comprovadas pela certificação obtida em maturidade de processos.

A K\&M, também de médio porte, constituiu um parâmetro para entendermos as limitações do processo inovativo associadas ao nicho de atuação de baixo valor agregado. Os serviços de TI, nesse caso, representam um conjunto de funções dirigidas primordialmente à reparação e manutenção de hardware, atividade que parece prescindir de níveis elevados de conhecimento, uma vez que mudanças explorando a dimensão conceitual estão incorporadas aos equipamentos. Por outro lado, a K\&M mostra que a construção prévia de capacitações habilita a empresa a participar de redes de inovação.

Pequenas empresas nacionais revelam um potencial criativo importante, em boa parte alimentado por tecnologias free e/ou open source (fundamentalmente TICii e TICgi). Em firmas desse porte, as inovações de conceito limitam-se a customizações, mas as de gestão, como a abertura de novos canais de prestação remota de serviços e mudanças nas estratégias de negócios, contribuem para a diversificação e especialização (casos da WM e Clavis).

As duas corporações transnacionais entrevistadas, Alpha e Unisys, dispõem de um grande arsenal de ferramentas digitais, condição assegurada por contratos globais com outras grandes empresas como a Microsoft. Entretanto, estratégias de inovação definidas de modo centralizado, ao concentrarem atividades mais intensivas em conhecimento nas unidades localizadas em outros países, pareceram ditar o ritmo e a direção das atividades de inovação no Brasil, limitando tanto o uso de TICdi mais sofisticadas (exemplo: para design de alto nível) quanto o surgimento de inovações de serviço (notadamente em termos de opções tecnológicas e de conceito).

Não obstante, podemos realçar, como um enorme diferencial a favor das ETN, o acesso a um conjunto de ferramentas para prospecção de informação, integração de redes e sistemas, e e-learning, que compõem uma base interna mundial de conhecimento, alimentando potencialmente todas as atividades de inovação.

\section{(II) Aprendizado}

Atividades de inovação intensivas em conhecimento normalmente se apoiam em processos de aprendizado que transcendem o simples "aprender fazendo", pois se trata de empresas localizadas na fronteira tecnológica que precisam investir maciçamente em atividades de P\&D. Isso, contudo, pareceu algo distante das rotinas de todas as firmas pesquisadas, sobretudo no que tange a processos de pesquisa (learning-by-research).

Atividades mais complexas de desenvolvimento constituem exceção (observada apenas na Marlin) e tiveram lugar dentro de estruturas predominantemente não formalizadas, o que, como vimos, é uma característica comum aos serviços. Ainda que não tenha realizado atividades formais de pesquisa, a Marlin orientou boa parte de seus processos de aprendizado para inovação, envolvendo praticamente todas as modalidades dentro da taxonomia de Lundvall (1988). As firmas menores (FPM, WM e Clavis) aprendem por meio da execução de tarefas, cujo foco recaiu em programação e customização de serviços (atividade de implementação). 0 único caso de learning-by-using foi registrado na empresa $K \& M$, no contexto da parceria com a Marlin. 
O treinamento foi uma modalidade intensa nas ETN, sem ter, todavia, um direcionamento específico para o uso de TIC visando gerar inovações. Além disso, grande parte do treinamento foi self-study, ou seja, funcionários acessam material de cursos nas bases digitais de conhecimento e aprendem sozinhos com suporte de ferramentas de e-learning. Vale destacar, nas ETN, o aprendizado por interação com o suporte de TIC, que ocorre internamente às corporações. Já a inovação aberta não compõe o leque de estratégias da Alpha e da Unisys, pelo menos no Brasil.

(III) Mudanças organizacionais de suporte ao uso de TIC

A análise realizada revela, primeiramente, que inovações organizacionais apenas repercutiram sobre o processo de inovação em empresas de médio (K\&M e Marlin) e grande porte (Alpha e Unisys). Nesses casos, a mudança na cultura corporativa e a adjacente postura de aceitação de novas tecnologias exerceram um papel significativo. Inovações de gestão foram mais abrangentes em duas empresas em particular, pois envolveram aspectos complementares como demissões, treinamento de diretores e redução de camadas hierárquicas.

O fato de a empresa Alpha não ter registrado mudanças organizacionais expressivas para dar suporte ao uso de TIC não implica passividade em relação à inovação de gestão. Esta ocorre predominantemente por meio de uma trajetória de aprendizado organizacional que sistematicamente aperfeiçoa rotinas e procedimentos e conduz um processo dinâmico de consolidação de estruturas globais de gestão.

A literatura e os surveys de inovação apontam que a informalidade, a falta de estrutura e organização, e a fragilidade dos fluxos de informação e conhecimento são traços característicos da inovação que podem ser comuns em países em desenvolvimento e em determinados segmentos do setor de serviços (Miranda \& Figueiredo, 2010). No caso das micro e pequenas empresas estudadas, pareceu não haver capacitação de gestão suficiente para introdução de inovações organizacionais que, pelo menos em tese, precisariam estar sempre ocorrendo para habilitar outras inovações.

(IV) Barreiras ao uso de TIC no suporte à inovação

No tocante ao ambiente interno às firmas, podemos destacar a rigidez organizacional como uma barreira significativa apontada por muitas empresas, à exceção das menores, sendo o principal ponto nesse âmbito o comportamento de aversão ao uso de novas tecnologias. As ETN demonstraram maior capacidade para superar isso (com auxílio de equipes de gestão de mudança, por exemplo) e revelaram, como outro fator de rigidez, a falta de autonomia das subsidiárias brasileiras para realizar atividades mais intensivas em conhecimento. Há, contudo, dife- renças consideráveis dependendo da linha de negócios das ETN, pois existem áreas que oferecem melhores oportunidades.

Os principais problemas internos defrontados pelas PMEs são a falta de competências e capacitações de gestão, que dificultam inovações organizacionais, e falta de pessoal técnico qualificado, tanto para exercer atividades mais simples, como programação, quanto para tarefas mais criativas, como design. Pequenas e médias empresas nacionais tendem a sofrer mais com a alta rotatividade de funcionários, devido à incapacidade de pagar salários comparáveis às ETN e oferecer possibilidades de realização de tarefas mais criativas.

Em relação ao ambiente externo, o custo das licenças relativas à propriedade intelectual constitui o obstáculo mais significativo, especialmente para quem tem grandes empresas usuárias de ferramentas proprietárias mais sofisticadas como cliente. Empresas que atendem usuários de menor porte contornam tais dificuldades por meio do uso de ferramentas free e/ou open source.

A maior e mais presente barreira (em todas as firmas pesquisadas) foi a falta de pessoal qualificado no mercado, a qual, em algumas entrevistas, foi vinculada parcialmente a deficiências no ensino universitário. Essa foi uma grave questão, que já havia sido apontada, possuindo uma série de nuances, pois os perfis de qualificação exigidos tenderam a variar conforme nossos recortes.

Empresas menores manifestaram grande necessidade de pessoas com competência em programação, dado que se concentraram em atividades mais repetitivas. A empresa Marlin, por outro lado, ao enfrentar uma competição fortemente baseada em inovações de conceito, encontrou grande dificuldade em contratar pessoas aptas a lidar com ferramentas mais sofisticadas de desenvolvimento/design. Uma maneira de competir com as multinacionais no mercado de trabalho é oferecer ações da empresa e participação nos lucros para atrair profissionais de nível mais elevado. As ETN igualmente encontraram dificuldades nesse âmbito, todavia assinalaram uma barreira não apontada pelas demais empresas: a grande escassez de profissionais com proficiência na língua inglesa.

\section{CONCLUSÕES}

A pesquisa realizada em empresas brasileiras de software constatou que as inovações habilitadas por TIC não constituem eventos isolados e pontuais, uma vez que alteram processos, formas de relacionamento com parceiros e clientes e contribuem para uma maior integração de funções organizacionais. Observamos diferenças importantes em termos de inovação em função do porte, 
nacionalidade e segmento em que a firma opera. As grandes empresas multinacionais contam com um conjunto abrangente e sofisticado de ferramentas para prospecção de informação, integração de redes e sistemas e e-learning, graças a seu acesso à base interna mundial de conhecimento da corporação.

A falta de autonomia das subsidiárias brasileiras para realizar atividades mais intensivas em conhecimento constitui, entretanto, um fator limitante para o desenvolvimento local de inovações mais sofisticadas. Já as pequenas e microempresas nacionais revelam um uso mais restrito das ferramentas, limitando-se, em geral, a adaptar programas às necessidades de seus clientes. Nessas empresas, o acesso a novas tecnologias free e/ou open source vem se revelando crítico para fomentar uma maior diversificação e especialização. Um problema estrutural, contudo, mostrou-se transversal às firmas investigadas: a falta de pessoal qualificado para lidar com ferramentas mais sofisticadas de desenvolvimento/design. A insuficiente proficiência em inglês no Brasil afeta principalmente as empresas de maior porte, enquanto empresas menores revelam dificuldades na contratação e retenção de programadores, diante da concorrência com grandes empresas na atração de talentos. Empresas que atuam em segmentos mais intensivos em conhecimento destacam-se pela capacidade de utilizar TIC no suporte às três atividades de inovação (gestão, desenvolvimento e implementação), apoiadas na capacitação técnica e no maior direcionamento estratégico às atividades de inovação.

A segunda questão examinada neste artigo refere-se às tecnologias, aos canais habilitados pelas ferramentas digitais e aos processos de aprendizado para inovação. As organizações investigadas apresentam importantes diferenças nesses aspectos. As empresas de maior sucesso são capazes de conjugar várias ferramentas digitais visando suportar as atividades de gestão, implementação e desenvolvimento. Por meio da habilitação de múltiplos canais, é possível melhorar a integração interna e externa, a qualidade de produtos, a gestão de processos e aumentar a produtividade.

Em relação ao processo de aprendizagem, sabemos que as atividades de inovação intensivas em conhecimento normalmente se apoiam em atividades de P\&D. Entretanto, nas empresas de software entrevistadas, o processo de aprendizado ainda depende intensivamente do "aprender fazendo" e "aprender usando". Isso é particularmente visível nas firmas menores, que aprendem por meio da execução de tarefas e da customização de serviços. Nas empresas multinacionais, observa-se um processo de aprendizado centrado no treinamento contínuo, baseado principalmente em ferramentas de e-learning. Observa-se também o aprendizado por interação global por meio da troca de conhe- cimentos e experiências entre membros da corporação como um todo.

A terceira e última questão analisada aqui se refere às mudanças organizacionais necessárias para o uso de ferramentas mais avançadas de TIC. A análise revela que inovações organizacionais ocorrem essencialmente em empresas de médio e grande portes. A mudança na cultura corporativa, principalmente quanto à postura de aceitação de novas tecnologias, constitui uma condição essencial para que tais mudanças ocorram. A rigidez organizacional e o comportamento de aversão ao uso de novas tecnologias constituem barreiras significativas para a difusão de inovações. As empresas multinacionais demonstram maior capacidade para superar a rigidez por contar com o auxílio de equipes especializadas na gestão de mudanças. Já nas empresas de pequeno porte, a informalidade, a fragilidade dos fluxos de informação e conhecimento e a insuficiente capacitação em gestão impedem a introdução de inovações dessa natureza. Com relação ao ambiente externo, o alto custo das licenças e as barreiras levantadas pela propriedade intelectual foram significativas para empresas domésticas. A dificuldade de acesso a ferramentas proprietárias mais sofisticadas, exigidas para operar em determinadas redes, conduz as empresas a se limitarem a mercados que usam ferramentas free e/ou open source.

De uma maneira geral, as empresas entrevistadas revelaram capacidade para utilizar TIC como uma importante ferramenta de inovação, embora com diferenças nos resultados de acordo com o tamanho, origem do capital e nicho de atuação das organizações. Processos mais robustos envolveram a conjugação de diversas tecnologias, que, por meio da habilitação de vários canais, apoiaram atividades de inovação, mobilizando mudanças em termos de conceito, opções tecnológicas, interface com cliente, sistema de distribuição e de natureza organizacional.

\section{NOTA DE AGRADECIMENTO}

Os autores agradecem o apoio da CAPES.

\section{REFERÊNCIAS}

Abreu, M., Grinevich, V., Kitson, M., \& Savona, M. (2010). Policies to enhance the "hidden innovation" in services: Evidence and lessons from the UK. The Service Industries Journal, 30(11), 99-118. doi:10.1080/02642060802236160

Albertin, A., \& Albertin, R. (2005). Tecnologia de informação e desempenho empresarial: As dimensões de seu uso e sua relação com os benefícios de negócio. São Paulo: Atlas. 
Albertin, A., \& Albertin, R. (2007). Alinhamento estratégico da TI com o desempenho empresarial. In F. de Oliveira (Org.). Tecnologia da informação e comunicação: A busca de uma visão ampla $e$ estruturada (Cap. 2, pp. 40-46). São Paulo: Pearson Prentice Hall.

Antonelli, C. (2003). The digital divide: Understanding the economics of new information and communication technology in the global economy. Information Economics and Policy, 15(2), 173-199. doi:10.1016/S0167-6245(02)00093-8

Autor, D., Katz, L., \& Krueger, A. (1998). Computing inequality: Have computers changed the labor market? The Quarterly Journal of Economics, 113(4), 1169-1213. doi:10.1162/003355398555874

Black, S., \& Lynch, L. (1997). How to compete: The impact of workplace practices and information technology on productivity [Working Paper n. 6120]. National Bureau of Economic Research.

Brynjolfsson, E. (1993, Dezembro). The productivity paradox of information technology. Magazine Communications of the ACM. Recuperado de http://cacm.acm.org/

Brynjolfsson, E., \& Hitt, L. (2003). Computing productivity growth: Firmlevel evidence. The Review of Economics and Statistics, 85(4), 793808. doi:10.1162/003465303772815736

Brynjolfsson, E., \& Saunders, A. (2010). Wired for Innovation: How information technology is reshaping the economy. Cambridge: The MIT Press.

Bueno, B., \& Balestrin, A. (2012). Inovação colaborativa: Uma abordagem aberta no desenvolvimento de novos produtos. RAERevista de Administração de Empresas, 52(5), 517-530.

Carr, N. (2003). IT doesn't matter. Harvard Business Review. Recuperado de https://hbr.org/

Chen, M., Nunamaker, J., \& Weber, E. (1989). Computer-aided software engineering: Present status and future directions. ACM SIGMIS Database, 20(1), 7-13. doi:10.1145/71232.71234

Chesbrough, H. (2011, Dezembro). Bringing open innovation to services. MIT Sloan Management Review, 52(2). Recuperado de http:// sloanreview.mit.edu/

Djellal, F., \& Gallouj, F. (1999). Services and the search for relevant innovation indicators: A review of national and international surveys. Science and Public Policy, 26(4), 218-232. doi:10.3152/147154399781782400

Dodgson, M., Gann, D., \& Salter, A. (2005). Think, play, do: Technology, innovation and organization. New York: Oxford Univ. Press.

Falk, M. (2001). Diffusion of information technology, internet use, and the demand for heterogeneous labor [Discussion Papers n. 1-48]. Center for European Economics.

Gago, D., \& Rubalcaba, L. (2006). Innovation and ICT in service firms: Towards a multidimensional approach for impact assessment. Journal of Evolutionary Economics, 17(1), 25-44. doi:10.1007/s00191006-0030-8

Gallouj, F., \& Savona, M. (2010). Towards a theory of innovation in service: A state of the art. In F. Gallouj \& F. Djellal (Eds.). The handbook of innovation and services: A multi-disciplinary perspective. Aldershot: Edward Elgar.

Giovanetti, B., \& Menezes-Filho, N. (2003). Tecnologia e demanda por qualificação na indústria brasileira. RBE-Revista Brasileira de Economia, 57(3), 569-603. doi:10.1590/s0034-71402003000300004

Green, L., \& Miles, I., \& Rutter, J. (2007). Hidden innovation in the creative sectors. Manchester Institute of Innovation Research, Working Paper for NESTA.

Greenan, N., \& Mairesse, J. (1996). Computers and productivity in France: Some evidence [Working Paper n. 5836]. National Bureau of Economic Research.

Hertog, P. Den. (2000). Knowledge-intensive business services as co-producers of innovation. International Journal of Innovation Management, 4(4), 491-528. doi:10.1142/s136391960000024X

Hipp, C., \& Grupp, H. (2005). Innovation in the service sector: The demand for service-specific innovation measurement concepts and typologies. Research Policy, 34(4), 517-535. doi:10.1016/j. respol.2005.03.002

Licht, G., \& Moch, D. (1999). Innovation and information technology in services. Canadian Journal of Economics, 32(2), 363-383. doi:10.2307/136427

Lundvall, B-A. Innovation as an interactive process: From user-producer interaction to the National System of Innovation (1988). In: G. Dosi, C. Freeman, R. Nelson, G. Silverberg \& L. Soete (Eds.). Technical change and economic theory. London: Pinter Publishers, chp. 11, p. 163-181.

Lynch, L. (2004). Reorienting training policies to meet the challenges of information and communication technologies. In L. Paganetto (Ed.). Knowledge economy, information technologies and growth (Chp. 11, pp. 319-334). Hampshire: ASHGATE.

Mendonça, M., Freitas, F., \& Souza, J. (2009). Tecnologia da informação e produtividade na indústria brasileira. RAE-Revista de Administração de Empresas, 49(1), 74-85.

Miles, I., \& Ducatel, K. (2005). Innovation in services. In J. Fagerberg, D. Mowery, \& R. Nelson. The Oxford handbook of innovation (Chp. 16, pp. 433-458). New York: Oxford Univ. Press.

Miles, I. (2008). Patterns of innovation in service industries. IBM Systems Journal, 47(1), 115-128.

Miranda, E., \& Figueiredo, P. (2010). Dinâmica da acumulação de capacidades inovadoras: Evidências de empresas de software no Rio de Janeiro e em São Paulo. RAE-Revista de Administração de Empresas, 50(1), 75-93.

Nambisan, S. (2013). Information technology and product/service innovation: A brief assessment and some suggestions for future research. Journal of the Association for Information Systems, 14(Special Issue), 215-226.

Oliveira, P., \& Hippel, E. Von. (2011, July). Users as service innovators: The case of banking services. Research Policy, 40(6), 806-818. doi:10.1016/j.respol.2011.03.009

Paganetto, L., Becchetti, L., \& Bedoya, D. (2004). The impact of IT investment on productivity and efficiency. In L. Paganetto (Ed.). Knowledge economy, information technologies and growth (Chp. 8, pp. 215-266). Hampshire: ASHGATE.

Sanchez, O., \& Albertin, A. (2009). A racionalidade limitada nas decisões de investimento em Tecnologia da Informação. RAE-Revista de Administração de Empresas, 49(1), 86-106.

The Organisation for Economic Co-operation and Development. (2005). Guidelines for collecting and interpreting innovation data - Oslo Manual. Paris: OECD.

Toinoven, M., \& Tuominen, T. (2007). Emergence of innovation in services. Service Industries Journal, 29(7), 887-902. doi:10.1080/02642060902749492 